\title{
Antikoagulation und Regionalanästhesie - neue ESAIC-Leitlinie
}

Univ.-Prof. Dr. Thomas Volk stellt rezente Empfehlungen der European Organisation for Anaesthesia, Intensive Care, Pain and Perioperative Medicine (ESAIC) vor.

Ü bergeordnetes Ziel der DGAI- und ESAIC-Guidelines ist die Vermeidung von epiduralen Blutungen im Rahmen der rückenmarksnahen Regionalanästhesie. Hinsichtlich der Vorgehensweise sei es interessant zu wissen, dass die Grundidee der beiden Fachgesellschaften jeweils eine andere war, erläutert Univ.Prof. Dr. Thomas Volk, Direktor der Klinik für Anästhesiologie, Intensivmedizin und Schmerztherapie in Homburg.

Die Pharmakokinetik-Krücke ist jene, mit der im Wesentlichen die DGAI argumentiert. Rosencher et al. gehen davon aus, dass bei angegebener terminaler Halbwertszeit und niedriger Antikoagulanziendosis nach

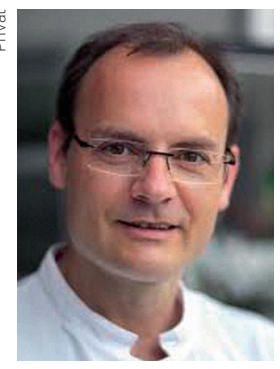

Univ.-Prof. Dr.

Thomas Volk

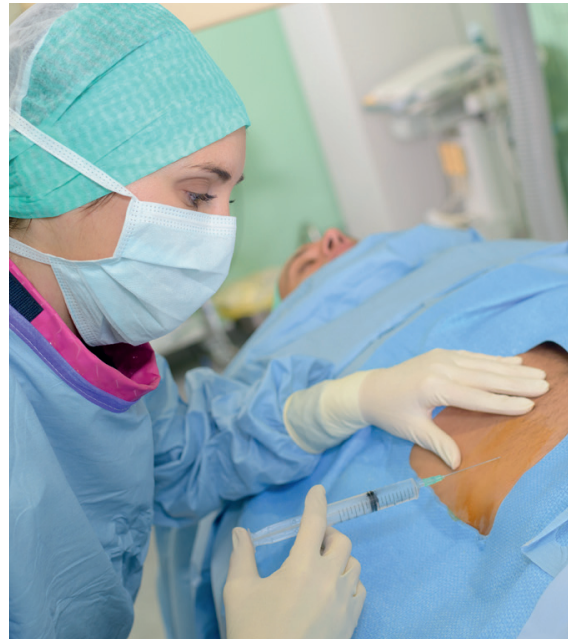

tiefen Nervenblockaden. Oberflächliche bleiben im Fall einer Blutung in aller Regel ohne Konsequenzen, sind leicht erkennund behandelbar. Sie können deswegen ohne Pausierung der Antikoagulation durchgeführt werden. Tiefe Blockaden sind hingegen schlecht komprimierbar und schwierig zu erkennen, eine Behebung ist selbst bei zeitnaher Diagnose aufwendig. „Diese tiefen Nervenblockaden, von denen möchten wir, dass sie behandelt werden wie neuraxiale", betont der Experte. Gleiches gelte im Übrigen auch für die Geburtshilfe, wobei laut ESAIC in besonderen Fällen von dieser Empfehlung abgewichen werden kann, etwa bei signifikant erhöhtem Thromboembolierisiko oder dringenden Eingriffen.

\section{AUSGEWÄHLTE ANTIKOAGULANZIEN}

Während die DGAI-Leitlinie bei Vitamin-KAntagonisten keine Angaben zu Pausenzeiten vor elektiven OPs macht, schlägt die ESAIC für Acenocoumarol drei Tage, für Warfarin fünf Tage und für Phenprocoumon sieben Tage vor. Auch der anzustrebende INR-Wert differiert zwischen den beiden Guidelines. So empfiehlt die DGAI generell einen INR $\leq 1,4$, wohingegen die ESAIC Normwerte anstrebt beziehungsweise ein INR $<1,5$ akzeptabel sein kann. Bei den Heparinen besteht der Unterschied darin, dass ESAIC auf zeitliche Korridore verzichtet (z.B. DGAI: Fondaparinux 36-42 h, ESAIC: $36 \mathrm{~h}$ ).
Die Handhabung direkter oraler Antikoagulanzien (DOAK) ist etwas komplexer. Prof. Volk kommt hier insbesondere auf die gelebte Praxis des tageweisen Einnahmestopps zu sprechen und weist auf dessen Unzulänglichkeit in Bezug auf Einnahmehäufigkeit und Zeitpunkt hin. Entspricht ein Tag Unterbrechung wirklich 24 Stunden? Schließlich kommen noch die Nacht und der OP-Tag hinzu. Bei der zweimal täglichen Einnahme wären dies effektiv immerhin 36 Stunden, bei einmal morgendlicher sogar 48. Diesem Gedanken folgend vereinfacht die ESAIC das Schema der DGAI und rät grundsätzlich bei erhaltener Nierenfunktion für alle vier DOAKs zu einem Einnahmestopp von 72 Stunden vor der geplanten OP. „Das übersetzt sich in zwei Tage Pause und am dritten Tag vor der OP wird zum Frühstück die letzte Dosis eingenommen", zeigt sich Prof. Volk zufrieden.

Bezüglich der Thrombozytenaggregationshemmer ASS, Clopidogrel, Prasugrel und Ticagrelor finden sich prinzipiell keine Änderungen. Allerdings gibt es eine Stellungnahme zu dem Problem, falls eine akute Koronarintervention bei Patient*innen mit rückenmarksnahem Katheter notwendig wird. Wenn ASS/Clopidogrel und die Antikoagulanzien noch nicht gegeben wurden, ist eine Katheter-Entfernung möglich. Andernfalls muss mit kurzwirksamen Substanzen für eine Antikoagulationslücke unter Aktivitätsmessung der antithrombozytären Wirkung gesorgt werden, wobei der Katheter in dieser Lücke herausgenommen wird. Für niedermolekulares Heparin und Fondaparinux ist eine anti-Xa-Aktivität von $\leq 0,1 \mathrm{IU} / \mathrm{ml}$ und für DOAKs eine Plasmakonzentration < $30 \mathrm{ng} /$ $\mathrm{ml}$ vorgesehen.

Bericht: Mag. Christopher Waxenegger Referenz:

Rosencher et al. Anaesthesia 2007; 62(11):1154-1160

Quelle: 23. Hauptstadtkongress der Deutschen Gesellschaft für Anästhesiologie und Intensivmedizin (DGAI); Symposium: Leitlinien auf dem Bierdeckel, 16.-17. September 2021, Berlin (digital) 